\title{
Friedel Crafts Acylation of Anisole With Modified Zeolites
}

\author{
Sreedhar Inkollu ${ }^{*}$, Mohammed Shoebuddin Habeeb ${ }^{1}$ and Sreenivasulu Bolisetty ${ }^{1}$ \\ ${ }^{1}$ Department of Chemical Engineering, BITS-Pilani, Hyderabad Campus, Hyderabad, India
}

\begin{abstract}
Acylation of anisole is an industrially significant reaction in the synthesis of various fine chemicals, agrochemicals, pharmaceuticals and fragrances. It has been conventionally carried out using the catalysts like $\mathrm{AlCl}, \mathrm{FeCl} 3, \mathrm{ZnCl3}, \mathrm{HF}$ etc which suffer from major drawbacks like large consumption of chemicals, associated corrosion, safety, disposal and material handling issues besides lower conversions and selectivities. Hence the conventional catalysts have been replaced with solid acid catalysts like H Beta, H ZSM5 etc to overcome the above said drawbacks. In this work, liquid phase acylation of anisole has been carried out employing an effective zeolite H ZSM 5 modified with HPW and NaOH. Macrolevel process standardization has been done with reference to various critical parameters like temperature, reaction time, molar ratio of reactants, calatyst amount for both types of the catalysts. It has been found that though both performed well in achieving high conversions, HPW modified catalyst was relatively better.
\end{abstract}

Keywords: Acylation of anisol, modified zeolites, process standardization, kinetics, Langmuir Hinshelwood model

\section{Introduction}

Acylation of aromatic compounds is one of the vital reactions for producing aromatic acylated ketones which were used in the manufacture of pharmaceuticals like Ibuprofen, fine chemicals, fragrances and agrochemicals [1-4]. The use of traditional Lewis acid type catalyst like $\mathrm{AlCl}_{3}, \mathrm{FeCl}_{3}$ and anhydrous metal halides and bronsted acid catalysts like HF, polyphosphoric acid, sulfuric acid etc lead to undesirable products thereby being environmentally non-benign with serious waste disposal, corrosion and material handling issues. This conventional route also proved to be economically non-viable due to excess amounts of reactants required beyond the stoichiometric amounts leading to expensive regeneration and energy intensive downstream processing. This has led many scientist to explore novel and effective catalysts viz., H-Beta, H ZSM-5, H Y as they are green and cost effective due to higher conversions, selectivity's and stabilities with simple regeneration of the used catalysts [5-11]. Heteropolyacids were also found to be potential catalysts due to their high acidity and compatibility to various support materials to enhance their activity [12]. Also, the replacement of acid halides with carboxylic acids and/or anhydrides as acylating agents is crucial from the environmental viewpoint. Challenges still exist in commercializing this green technology in identifying better and cost effective catalysis with controllable properties and by overcoming the major impediment of rapid catalyst fouling leading to its deactivation.

In the present work, acylation of anisole leading to the formation of a mixture of 4- Methoxyacetophenone (desired) a 4-Methoxyacetophenone (undesired) along with acetic acid was carried out using acetic anhydride as the acylating agent in the presence of modified H-ZSM 5 catalyst. The catalyst has been modified by two methods i.e using 0.2M alkali solution $(\mathrm{NaOH})$ and by impregnating with HPW (Tungstophosphoric acid) to see if there is any synergistic influence on its performance. The process has been standardized with reference to critical parameters like molar ratio of reactants, temperature, reaction time, catalyst modification protocol and quantity. Kinetic studies have also been conducted at the optimal conditions to identify the kinetic model and the relevant parameters. 


\section{Experiment}

\subsection{Chemicals}

Anisole and acetic anhydride were commercial samples procured from Merck and were used without further purification. The catalyst $\mathrm{H} \mathrm{ZSM}-5$ zeolite $(\mathrm{Si} / \mathrm{Al}=80), \mathrm{NaOH}$ and $\mathrm{HPW}$ were commercial samples procured from Sud Chemies India Pvt Ltd.

\subsection{Experimental Procedure and Set-up}

The liquid phase acylation of anisole was carried in a two necked glass reactor of volume $100 \mathrm{ml}$ at atmospheric pressure. The reaction mixture containing anisole and acetic anhydride together with zeolite catalyst were taken in the reactor placed on a heating mantle equipped with a magnetic stirrer. A small amount of sample was withdrawn periodically for further analysis.

\subsection{Catalyst Modification}

\subsubsection{Modification with $\mathrm{NaOH}$}

The ZSM 5 catalyst was reacted with $0.2 \mathrm{M} \mathrm{NaOH}$ and slurry was prepared for 10 min of reaction. Then the slurry was put in a centrifuge to obtain back the modified catalyst. Then catalyst was heated for 1 hour in oven for drying.

\subsubsection{Modification with HPW}

HPW and ZSM-5 mixture was prepared with 10\%, $20 \%$ and $30 \%$ weight percentage of HPW. The mixture H-ZSM5 and HPW are mixed and slurry was prepared and passed to mixer where it was uniformly mixed for a very short time and then it was poured over a filter paper and filtered out. The obtained residue was then heated in oven where it was dried till powdery form is obtained.

\subsection{Analytical}

The product samples were analysed using Agilent make Gas Chromatograph (Model 7820), equipped with a HP-5 column fitted with a Flame Ionization Detector.

\section{Results and Discussion}

\subsection{Effect of Reaction Temperature}

The acylation of acylation reaction has been carried out at temperatures ranging from $60-120 \mathrm{oC}$ and conversion are estimated at each of them. The results for two catalysts are shown in Fig 1 and Fig 2.

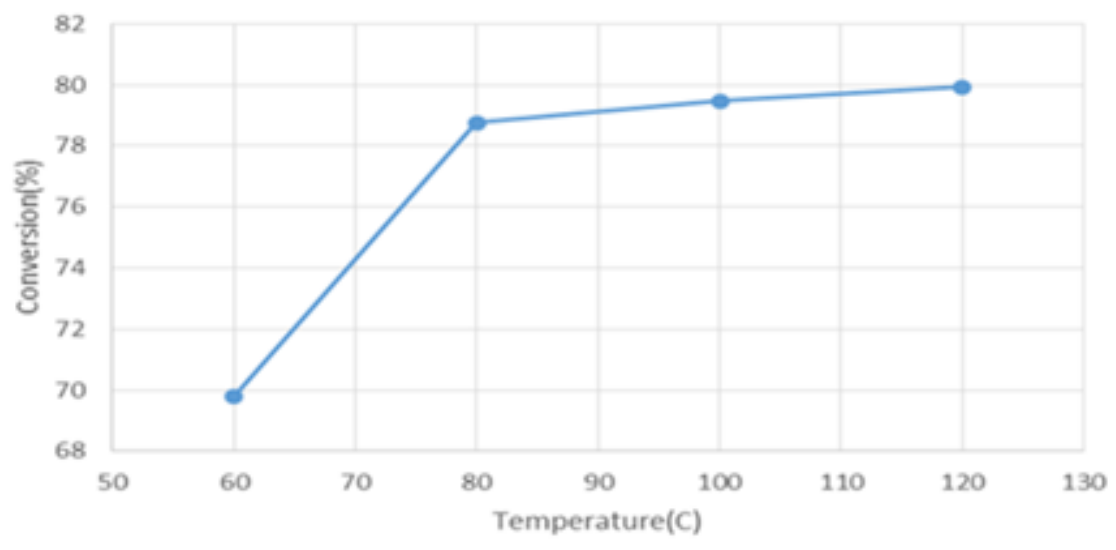

Fig.1. Effect of Temperature for production 4-methoxy-acetophenone using ZSM-5 modified with $0.2 \mathrm{~N} \mathrm{NaOH}$ (time 120 min, Weight of Catalyst 0.15 grams, Molar Ratio of Anisole to Acetic Anhydride 8:1) 


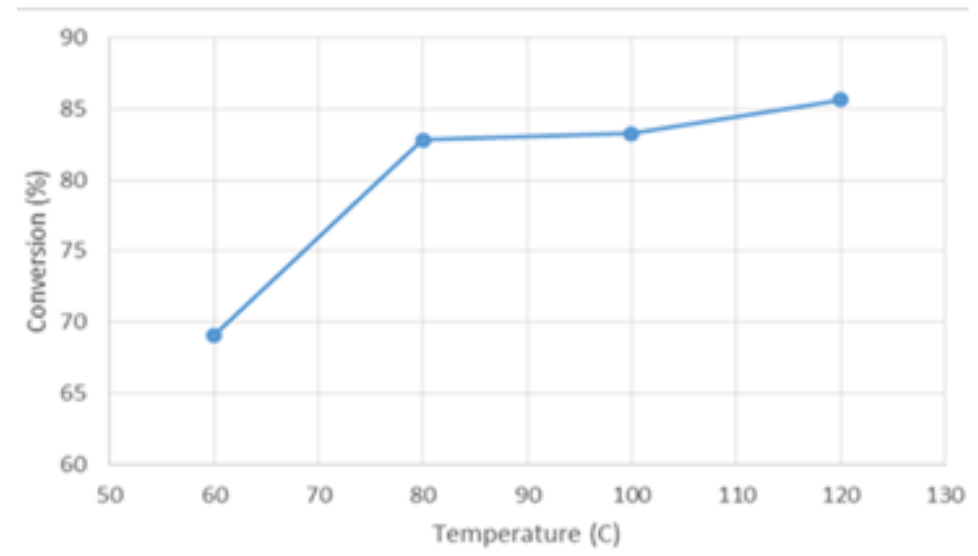

Fig 2. Effect of Temperature for production 4-methoxy-acetophenone using ZSM-5 impregnated with 30\% HPW (time $120 \mathrm{~min}$, Weight of Catalyst 0.15 grams, Molar Ratio of Anisole to Acetic Anhydride 8:1)

The product conversion was found to reach the maximum of $80 \%$ and $85 \%$ respectively at $100 \mathrm{oC}$ for both the catalysts. This might be due to the inhibiting effect of 4-methoxyacetophenone which could be strongly adsorbed on catalyst at higher conversions. The results are in agreement with the earlier reported results using other catalyst [11-14]. There were reports that even for acylation of toluene with acetic anhydride, similar trends were observed due to strong adsorption of methyl acetophenones [8].

\subsection{Effect of Nature of Catalyst}

H ZSM-5 was modified by impregnating with HPW at loading levels of 10\%, 20\% and 30\% which have then been used for carrying out acylation of anisole at 100oC. Fig. 3 below shows the results of the study. Initially percentage conversion decreased with increased loading, but then increased to a maximum of $84 \%$ at $30 \%$ which might be due to the some synergetic effect of weight ratio [15]. It has been reported that the HPW protonates the anhydride molecule generating an adsorbed acylium ion that subsequently attacks the $\pi$-electrons of anisole to form methoxyacetophenone [16,17]. From these figures, it also could be understood that both the modified catalysts performed well and impregnation of HPW on the catalyst has a relatively better influence on catalyst performance. when compared to loading with $0.2 \mathrm{~N} \mathrm{NaOH}$. The positive effect of $\mathrm{NaOH}$ could be attributed to the reduction of acidity of catalyst besides increasing catalyst activity by reducing fouling. Our results are qualitatively in line with those reported on acylation of anisole using HBeta and Silica catalysts loaded with HPWs [15-18].

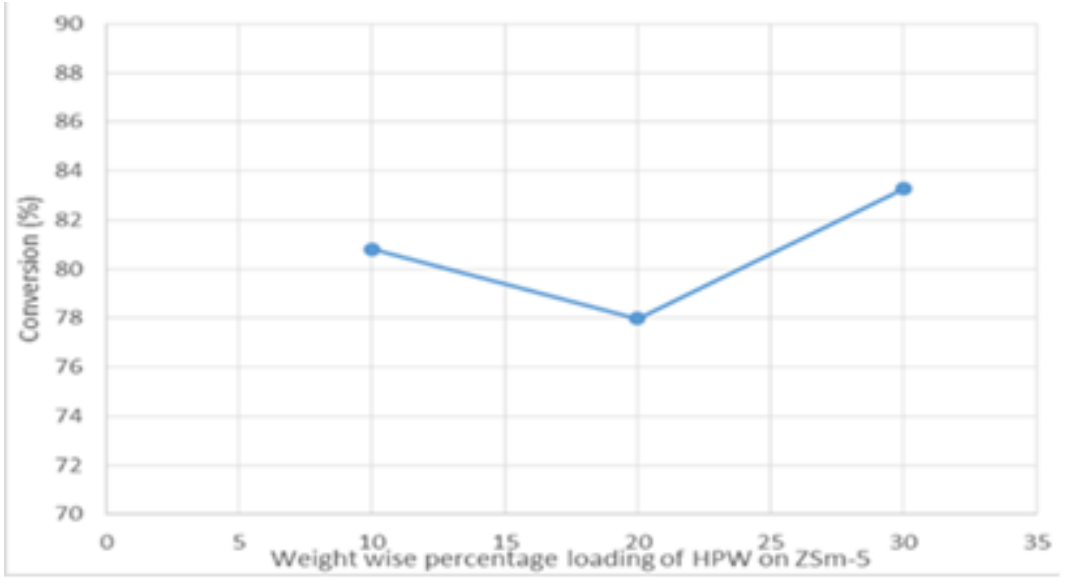

Fig. 3. HPW impregnated ZSM-5 conversion at different loading rate (time $120 \mathrm{~min}$, Weight of Catalyst $0.15 \mathrm{~g}$, Molar Ratio of Anisole to Acetic Anhydride 8:1, Temperature 100oC) 


\subsection{Effect of Catalyst Quantity}

Experiments have been carried out using various amounts of catalysts from $0.1 \mathrm{~g}$ to $0.6 \mathrm{~g}$ to study the influence of catalyst loading. For both the catalysts modified viz., HZSM-5 modified with $0.2 \mathrm{~N} \mathrm{NaOH}$ and HPW, it was found that there was an optimum quantity $(0.45 \mathrm{~g})$ that gave maximum conversions more than $90 \%$ beyond which they were found to decrease. Fig. 4 and 5 below gives the results of the study.

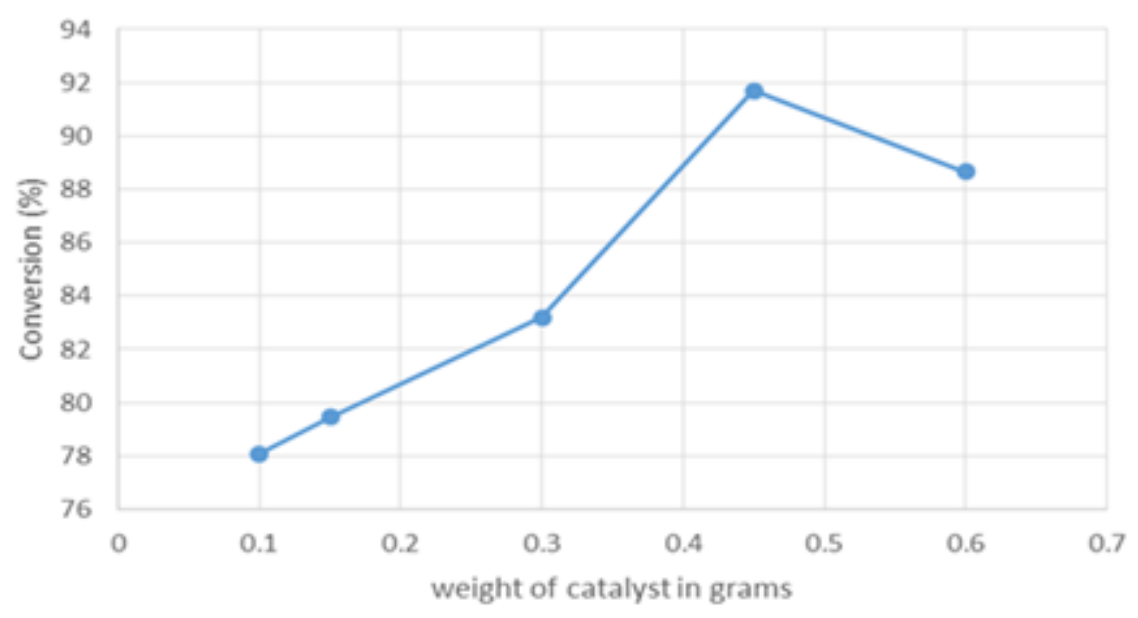

Fig.4. Effect of Catalysis quantity for production 4-methoxy-acetophenone using ZSM-5 modified with $0.2 \mathrm{~N} \mathrm{NaOH}$ (time $120 \mathrm{~min}$, temperature $100^{\circ} \mathrm{C}$, Molar Ratio of Anisole to Acetic Anhydride 8:1.

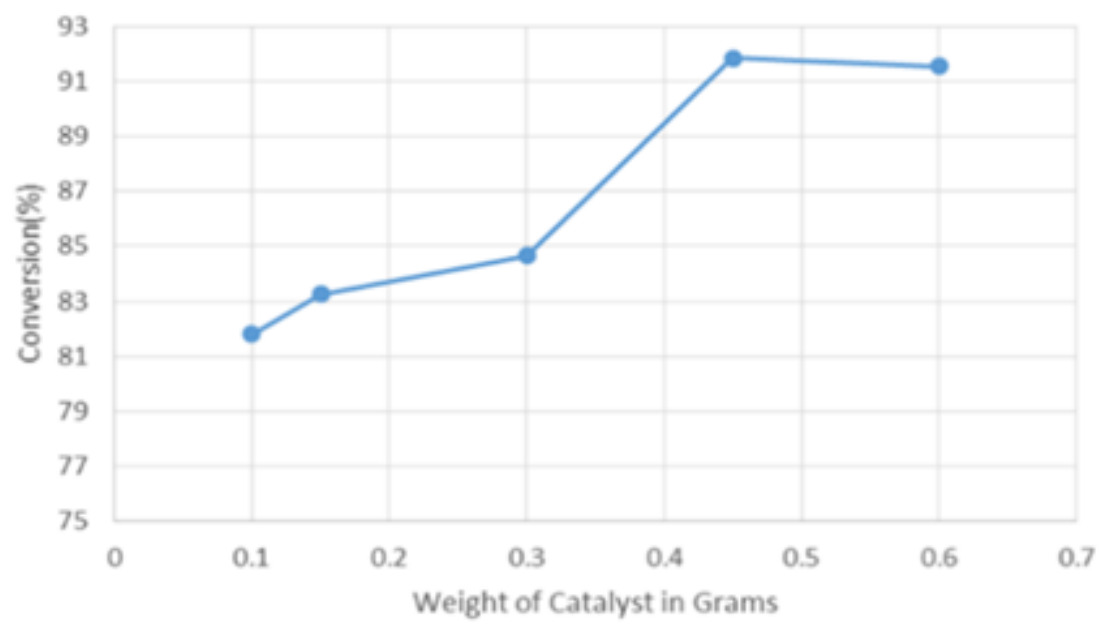

Fig.5 Effect of Catalysis quantity for production 4-methoxy-acetophenone using ZSM-5 impregnated with HPW (time 120 min, Temperature $100^{\circ} \mathrm{C}$, Molar Ratio of Anisole to Acetic Anhydride 8:1, HPW loading percentage 30\%)

The increase in conversion with the increase in catalyst amount could be explained due to the increase in the number of active sites with catalyst amount and hence the increase in conversion. But beyond certain amount, the additional active sites do not increase the adsorption of reactants at a given concentration. Other factors that could be attributed for this trend could be catalyst deactivation by coking or reversible adsorption of products on the active acid sites $[18,19]$. This fact could be supported by the deactivation studies carried out by D.Rohan, C. Canaff [20]. 


\subsection{Effect of Reaction Time}

To understand the influence of reaction time on conversion, experiments were conducted for different time intervals ranging from 30min to 180min. Results were shown in Fig. 6 and Fig. 7 for the two catalyst types viz. modified with $\mathrm{NaOH}$ and HPW.

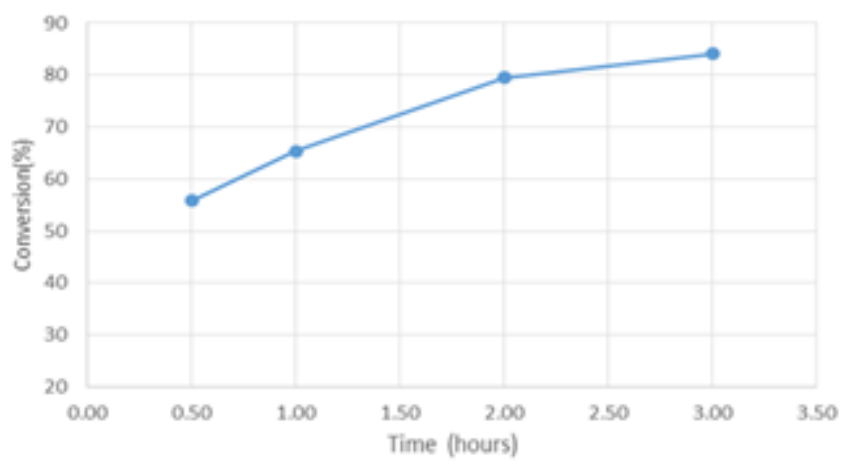

Fig.6. Effect of Reaction time for production 4-methoxy-acetophenone using ZSM-5 modified with $0.2 \mathrm{~N} \mathrm{NaOH}$ (weight of catalyst $0.15 \mathrm{~g}$, temperature $100^{\circ} \mathrm{C}$, Molar Ratio of Anisole to Acetic Anhydride 8:1).

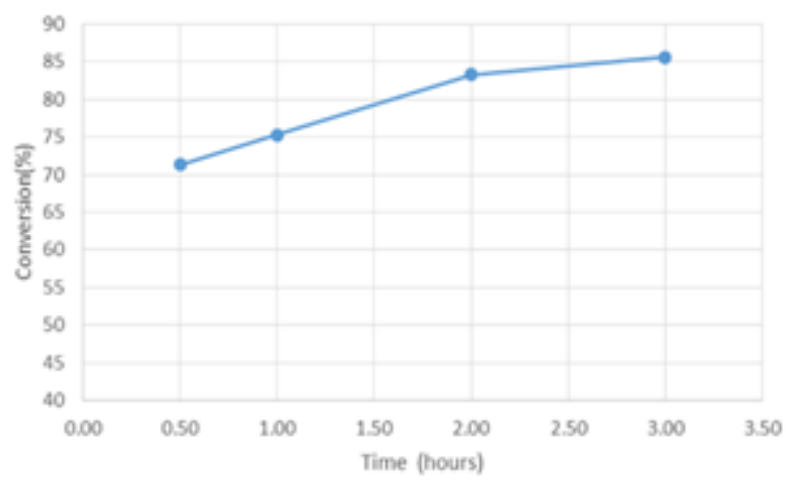

Fig.7. Effect of Reaction time for production 4-methoxy-acetophenone using ZSM-5 impregnated with HPW (time 120 min, Temperature $100^{\circ} \mathrm{C}$, Molar Ratio of Anisole to Acetic Anhydride 8:1, HPW loading percentage 30.

From the plots, it was evident that though conversion did increase with time, the rate of increase was more up till 2hours beyond which it got slowed down in both cases. This trend was more obvious in HPW modified catalyst where there was only marginal increase in conversion of $5 \%$ after 2 hours. Similar trends were reported too in the time range upto 3-4hours [11-14]. The reason for this trend could be equilibrium limitations of the reversible reaction and the catalyst deactivation due to coking or the reversible adsorption of products in the active sites of the catalyst making them unavailable for the reactants. Cardoso et al., [18] and Sarsani et al., [21] also reported that beyond 2 hours, the conversion increase in the acylation of anisole was only marginal employing HPW/Silica catalyst.

\subsection{Effect of Reactant Molar Ratio}

Experiments have been carried out employing various mole ratios of anisole to acetic anhydride ranging from 4:1 to 12:1 and the results are shown below in Fig 8 and Fig. 9. Though the conversion was found to increase with increasing mole ratio in both cases, it almost reached saturation at 8:1 for $\mathrm{NaOH}$ modified catalyst while in the case of the other catalyst modified with HPW, conversion was found to increase even beyond this mole ratio of 8:1 though at a slow rate. The increasing production of 4-Methoxy Acetophenone could be attributed to anhanced desorption of acetic acid and 4-methoxyacetophenone formed through the zeolite pores by excess anisole that acts as a self-solvent which would mean that products diffuse easily through the pores of the catalyst with effective washing by excess anisole [20]. 


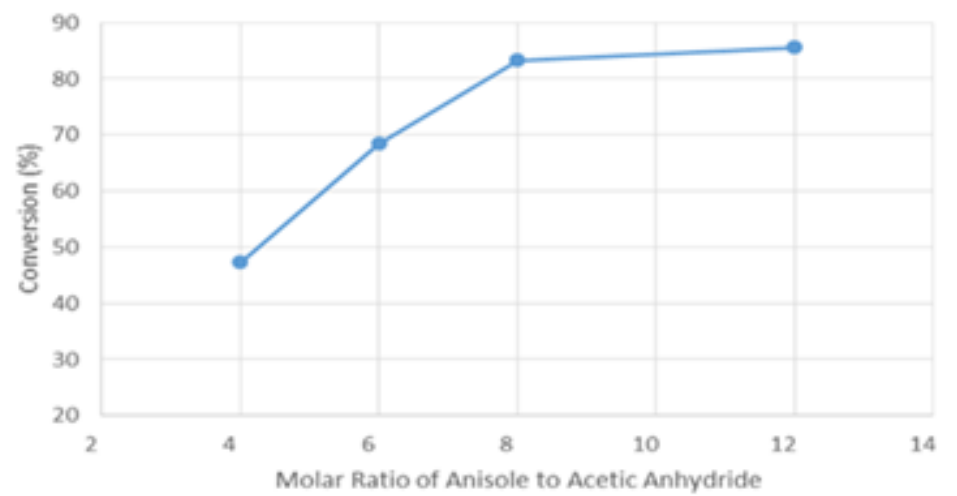

Fig.8. Effect of Molar ratio for production 4-methoxy-acetophenone using ZSM-5 modified with $0.2 \mathrm{~N} \mathrm{NaOH}$ (weight of catalyst $0.15 \mathrm{~g}$, temperature $100^{\circ} \mathrm{C}$, Time $120 \mathrm{~min}$ ).

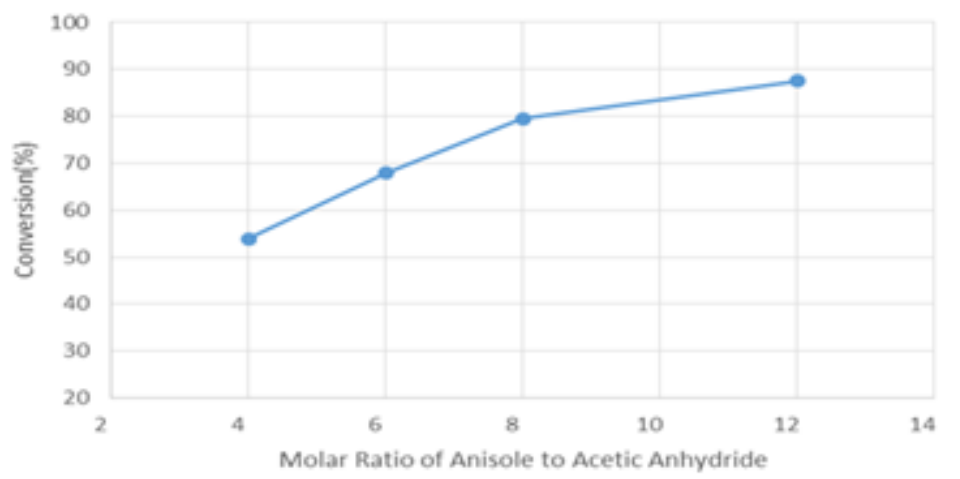

Fig.9. Effect of Molar Ratio for production 4-methoxy-acetophenone using ZSM-5 impregnated with HPW (time 120 min, Temperature $100^{\circ} \mathrm{C}$, Time $120 \mathrm{~min}$, HPW loading percentage 30).

\section{Conclusions}

The macro level standardization of the liquid phase acylation of anisole was done and the optimum set of parameters have been identified to be catalyst quantity of $0.15 \mathrm{~g}$, reaction time of $2 \mathrm{~h}$, temperature of $100^{\circ} \mathrm{C}$, molar ratio of anisole to acetic anhydride 8:1 were the optimal set of parameters for both the modifications of the catalyst. Relatively, HPW modification was found to give better results when compared to the modification with $\mathrm{NaOH}$.

\section{Acknowledgements}

The authors are delighted to express their gratitude to the management of BITS-Pilani, for funding and other contributions towards the research work.

\section{References}

[1] H.-G. Franck, J.W. Stadelhofer, Industrial Aromatic Chemistry: Raw Materials · Processes · Products, 1st ed., Springer-Verlag Berlin Heidelberg, Berline, 1988.

[2] H.W. Kouwenhoven, E.A. Gunnewegh, H. Van Bekkum, "Catalysis on solid acids and bases", in: J. Weitkamp, B. Lucke (Eds.), Proceedings 9601 of the DGMK Conference, Berlin, 14-15, March, 1996, DGMK, Hamburg, Hamburg, 1996: pp. 1-9.

[3] R.A. Sheldon, "Organic synthesis: past, present and future”, Chem.Ind., London, 1992.

[4] T. Fujisawa, K. Kondo, K. Sakai, “Process for preparing thiophene derivatives”, US4266067A, 1981. 
[5] G. Winé, C. Pham-Huu, M.-J. Ledoux, "Acylation of anisole by acetic anhydride catalysed by BETA zeolite supported on pre-shaped silicon carbide", Catalysis Communications. 7 (2006) 768-772.

[6] P. Moreau, A. Finiels, P. Meric, "Acetylation of dimethoxybenzenes with acetic anhydride in the presence of acidic zeolites", Journal of Molecular Catalysis A: Chemical. 154 (2000) 185-192.

[7] G.D. Yadav, M.S. Krishnan, "Solid acid catalysed acylation of 2-methoxy-naphthalene: role of intraparticle diffusional resistance", Chemical Engineering Science. 54 (1999) 4189-4197.

[8] P. Botella, A. Corma, J.M. López-Nieto, S. Valencia, R. Jacquot, “Acylation of Toluene with Acetic Anhydride over Beta Zeolites: Influence of Reaction Conditions and Physicochemical Properties of the Catalyst", Journal of Catalysis. 195 (2000) 161-168.

[9] Y. Ma, Q.L. Wang, W. Jiang, B. Zuo, "Friedal-Crafts acylation of anisole over zeolite catalysts", Applied Catalysis A: General. 165 (1997) 199-206.

[10] A. Corma, M. JoséCliment, H. García, J. Primo, "Design of synthetic zeolites as catalysts in organic reactions", Applied Catalysis. 49 (1989) 109-123.

[11] R. Selvin, B. Sivasankar, K. Rengaraj, "Kinetic studies on Friedel-Crafts acylation of anisole by clayzic", Reaction Kinetics and Catalysis Letters. 67 (1999) 319-324.

[12] J. Kaur, K. Griffin, B. Harrison, I. V Kozhevnikov, "Friedel-Crafts Acylation Catalysed by Heteropoly Acids", Journal of Catalysis. 208 (2002) 448-455.

[13] I. Sreedhar, H. Kantamneni, K. Suresh Kumar Reddy, K. V Raghavan, "Optimal process conditions for zeolite catalyzed acylation of anisole", Kinetics and Catalysis. 55 (2014) 229-232.

[14] R. Selvin, H.-L. Hsu, T.-M. Her, “Acylation of anisole with acetic anhydride using ZSM-5 catalysts: Effect of ZSM-5 particle size in the nanoscale range", Catalysis Communications. 10 (2008) 169-172.

[15] G. Bai, H. Zhang, T. Li, H. Dong, J. Han, "Friedel-Crafts acylation of anisole with hexanoic acid catalyzed by H $\beta$ zeolite-supported tungstophosphoric acid”, Research on Chemical Intermediates. 41 (2015) 5041-5048.

[16] K. Gaare, D. Akporiaye, "Modified zeolites as catalysts in the Friedel-Crafts acylation", Journal of Molecular Catalysis A: Chemical. 109 (1996) 177-187..

[17] I. Neves, F. Jayat, P. Magnoux, G. Pérot, F.R. Ribeiro, M. Gubelmann, M. Guisnet, “Acylation of phenol with acetic acid over a HZSM5 zeolite, reaction scheme", Journal of Molecular Catalysis. 93 (1994) 169-179.

[18] L.A.M. Cardoso, W.A. Jr, A.R.E. Gonzaga, L.M.G. Aguiar, H.M.C. Andrade, "Friedel-Crafts acylation of anisole with acetic anhydride over silica-supported heteropolyphosphotungstic acid (HPW/SiO2)", Journal of Molecular Catalysis A: Chemical. 209 (2004) 189-197.

[19] M.L. Kantam, K.V.S. Ranganath, M. Sateesh, K.B.S. Kumar, B.M. Choudary, "Friedel-Crafts acylation of aromatics and heteroaromatics by beta zeolite", Journal of Molecular Catalysis A: Chemical. 225 (2005) 15-20.

[20] D. Rohan, C. Canaff, E. Fromentin, M. Guisnet, “Acetylation of anisole by acetic anhydride over a HBEA zeoliteOrigin of deactivation of the catalyst", Journal of Catalysis. 177 (1998) 296-305.

[21] V.R. Sarsani, C.J. Lyon, K.W. Hutchenson, M.A. Harmer, B. Subramaniam, "Continuous acylation of anisole by acetic anhydride in mesoporous solid acid catalysts: Reaction media effects on catalyst deactivation", Journal of Catalysis. 245 (2007) 184-190. 